\title{
Factors of sums and alternating sums involving binomial coefficients and powers of integers
}

\author{
Victor J. W. Guo ${ }^{1}$ and Jiang Zeng ${ }^{2}$ \\ ${ }^{1}$ Department of Mathematics, East China Normal University, \\ Shanghai 200062, People's Republic of China \\ jwguo@math.ecnu.edu.cn, http://math.ecnu.edu.cn/ ${ }^{\sim}$ jwguo \\ ${ }^{2}$ Université de Lyon; Université Lyon 1; Institut Camille Jordan, UMR 5208 du CNRS; \\ 43, boulevard du 11 novembre 1918, F-69622 Villeurbanne Cedex, France \\ zeng@math.univ-lyon1.fr, http://math.univ-lyon1.fr/ ${ }^{\sim}$ zeng
}

Abstract. We study divisibility properties of certain sums and alternating sums involving binomial coefficients and powers of integers. For example, we prove that for all positive integers $n_{1}, \ldots, n_{m}$, $n_{m+1}=n_{1}$, and any nonnegative integer $r$, there holds

$$
\sum_{k=0}^{n_{1}} \varepsilon^{k}(2 k+1)^{2 r+1} \prod_{i=1}^{m}\left(\begin{array}{c}
n_{i}+n_{i+1}+1 \\
n_{i}-k
\end{array}\right) \equiv 0 \bmod \left(n_{1}+n_{m}+1\right)\left(\begin{array}{c}
n_{1}+n_{m} \\
n_{1}
\end{array}\right),
$$

and conjecture that for any nonnegative integer $r$ and positive integer $s$ such that $r+s$ is odd,

$$
\sum_{k=0}^{n} \varepsilon^{k}(2 k+1)^{r}\left(\left(\begin{array}{c}
2 n \\
n-k
\end{array}\right)-\left(\begin{array}{c}
2 n \\
n-k-1
\end{array}\right)\right)^{s} \equiv 0 \bmod \left(\begin{array}{c}
2 n \\
n
\end{array}\right),
$$

where $\varepsilon= \pm 1$.

Keywords: binomial coefficients, divisibility properties, Chu-Vandermonde formula, Lucas' theorem

AMS Subject Classifications: 05A10, 11B65, 11A07

\section{Introduction}

There have been lasting interests in binomial sums. Although some binomial sums have no closed formulas, it is still possible to show that they have some nice factors. For example, a result of Calkin [2] reads

$$
\sum_{k=-n}^{n}(-1)^{k}\left(\begin{array}{c}
2 n \\
n+k
\end{array}\right)^{m} \equiv 0 \quad \bmod \left(\begin{array}{c}
2 n \\
n
\end{array}\right) \quad \text { for } m \geq 1
$$

Generalizing Calkin's result, Guo, Jouhet, and Zeng [8] proved, among other things, that

$$
\sum_{k=-n_{1}}^{n_{1}}(-1)^{k} \prod_{i=1}^{m}\left(\begin{array}{c}
n_{i}+n_{i+1} \\
n_{i}+k
\end{array}\right) \equiv 0 \bmod \left(\begin{array}{c}
n_{1}+n_{m} \\
n_{1}
\end{array}\right)
$$

for all $n_{1}, \ldots, n_{m} \geq 1$ and $n_{m+1}=n_{1}$. Recently, motivated by the moments of the Catalan triangle (see [3,11]), Guo and Zeng [10] were led to study some different binomial sums and proved the congruence

$$
2 \sum_{k=1}^{n_{1}} k^{2 r+1} \prod_{i=1}^{m}\left(\begin{array}{c}
n_{i}+n_{i+1} \\
n_{i}+k
\end{array}\right) \equiv 0 \bmod n_{1}\left(\begin{array}{c}
n_{1}+n_{m} \\
n_{1}
\end{array}\right)
$$


for all $n_{1}, \ldots, n_{m} \geq 1$ and $n_{m+1}=n_{1}$.

In this paper we will prove some divisibility properties of another kind of sums and alternating sums involving binomial coefficients and powers of integers. Let $\mathbb{N}$ denote the set of nonnegative integers and $\mathbb{Z}^{+}$the set of positive integers. One of our main results may be stated as follows.

Theorem 1.1. For all $n_{1}, \ldots, n_{m} \in \mathbb{Z}^{+}, n_{m+1}=n_{1}$, and $r \in \mathbb{N}$, there holds

$$
\sum_{k=0}^{n_{1}} \varepsilon^{k}(2 k+1)^{2 r+1} \prod_{i=1}^{m}\left(\begin{array}{c}
n_{i}+n_{i+1}+1 \\
n_{i}-k
\end{array}\right) \equiv 0 \bmod \left(n_{1}+n_{m}+1\right)\left(\begin{array}{c}
n_{1}+n_{m} \\
n_{1}
\end{array}\right),
$$

where $\varepsilon= \pm 1$.

Actually, we shall derive Theorem 1.1 from the following more general result.

Theorem 1.2. For all $n_{1}, \ldots, n_{m} \in \mathbb{Z}^{+}, n_{m+1}=n_{1}$, and $r \in \mathbb{N}$, there hold

$$
\begin{aligned}
& \sum_{k=0}^{n_{1}} k^{r}(k+1)^{r}(2 k+1) \prod_{i=1}^{m}\left(\begin{array}{c}
n_{i}+n_{i+1}+1 \\
n_{i}-k
\end{array}\right) \\
& \equiv 0 \bmod \left(n_{1}+n_{m}+1\right)\left(\begin{array}{c}
n_{1}+n_{m} \\
n_{1}
\end{array}\right) n_{1}^{\min \{1, r\}} n_{m}^{\min \left\{1,\left(\begin{array}{c}
r \\
2
\end{array}\right)\right\}} \\
& \sum_{k=0}^{n_{1}}(-1)^{k} k^{r}(k+1)^{r}(2 k+1) \prod_{i=1}^{m}\left(\begin{array}{c}
n_{i}+n_{i+1}+1 \\
n_{i}-k
\end{array}\right) \\
& \quad \equiv 0 \bmod \left(n_{1}+n_{m}+1\right)\left(\begin{array}{c}
n_{1}+n_{m} \\
n_{1}
\end{array}\right) n_{1}^{\min \{1, r\}} n_{m}^{\min \{1, r\}}
\end{aligned}
$$

Indeed, by the expansion

$$
(2 k+1)^{2 r}=\left(4 k^{2}+4 k+1\right)^{r}=\sum_{i=0}^{r}\left(\begin{array}{l}
r \\
i
\end{array}\right) 4^{i} k^{i}(k+1)^{i},
$$

it is clear that Theorem 1.2 infers Theorem 1.1.

Recently, Miana and Romero [12, Theorem 10] evaluated the moments $\Psi_{r}(n):=\sum_{k=0}^{n}(2 k+1)^{r} A_{n, k}^{2}$, where $A_{n, k}(0 \leq k \leq n)$ are the ballot numbers defined by

$$
A_{n, k}=\frac{2 k+1}{2 n+1}\left(\begin{array}{c}
2 n+1 \\
n-k
\end{array}\right)=\left(\begin{array}{c}
2 n \\
n-k
\end{array}\right)-\left(\begin{array}{c}
2 n \\
n-k-1
\end{array}\right) .
$$

By the formulas of $\Psi_{1}(n), \Psi_{3}(n)$ and $\Psi_{5}(n)$ (see [12, Remark 11]), we conjecture that $\Psi_{2 r+1}(n)$ is divisible by $\left(\begin{array}{c}2 n \\ n\end{array}\right)$. More generally, we have

Conjecture 1.3. For all $r \in \mathbb{N}$ and $n, s \in \mathbb{Z}^{+}$such that $r+s \equiv 1(\bmod 2)$, there holds

$$
\sum_{k=0}^{n} \varepsilon^{k}(2 k+1)^{r} A_{n, k}^{s} \equiv 0 \quad \bmod \left(\begin{array}{c}
2 n \\
n
\end{array}\right),
$$

where $\varepsilon= \pm 1$.

As a check, let $n=7$, for all $r \geq 0$ and $s \geq 1$ such that $r+s \equiv 1(\bmod 2)$, the sum

$$
\sum_{k=0}^{7}(2 k+1)^{r} A_{7, k}^{s}=429^{s}+3^{r} 1001^{s}+5^{r} 1001^{s}+7^{r} 637^{s}+9^{r} 273^{s}+11^{r} 77^{s}+13^{r+s}+15^{r}
$$

is obviously divisible by $\left(\begin{array}{c}14 \\ 7\end{array}\right)=8 \times 3 \times 11 \times 13$.

In this paper, we shall confirm Conjecture 1.3 in some special cases.

Theorem 1.4. The congruence (1.1) holds if $2 n+1$ is a prime power or $s=1$.

In the next three sections we shall provide the proof of Theorem 1.2 corresponding, respectively, to the cases $m=1, m=2$ and $m \geq 3$. We then prove Theorem 1.4 in Section 5. Finally we give some further consequences of Theorem 1.1 and related conjectures in Section 6 . 


\section{Proof of Theorem 1.2 for $m=1$}

Let

$$
\begin{aligned}
P_{r}(n) & :=\sum_{k=0}^{n}\left(\begin{array}{c}
2 n+1 \\
n-k
\end{array}\right) k^{r}(k+1)^{r}(2 k+1), \\
Q_{r}(n) & :=\sum_{k=0}^{n}(-1)^{k}\left(\begin{array}{c}
2 n+1 \\
n-k
\end{array}\right) k^{r}(k+1)^{r}(2 k+1) .
\end{aligned}
$$

The $m=1$ case of Theorem 1.2 may be stated as follows.

Theorem 2.1. For all $n \in \mathbb{Z}^{+}$and $r \in \mathbb{N}$, there hold

$$
\begin{aligned}
& P_{r}(n) \equiv 0 \quad \bmod (2 n+1)\left(\begin{array}{c}
2 n \\
n
\end{array}\right) n^{\min \{2, r\}} \\
& Q_{r}(n) \equiv 0 \quad \bmod (2 n+1)\left(\begin{array}{c}
2 n \\
n
\end{array}\right) n^{\min \{2,2 r\}}
\end{aligned}
$$

Proof of Theorem 2.1. We proceed by induction on $r$. For $r=0$, we have

$$
\begin{aligned}
& P_{0}(n)=(2 n+1) \sum_{k=0}^{n}\left(\left(\begin{array}{c}
2 n \\
n-k
\end{array}\right)-\left(\begin{array}{c}
2 n \\
n-k-1
\end{array}\right)\right)=(2 n+1)\left(\begin{array}{c}
2 n \\
n
\end{array}\right), \\
& Q_{0}(n)=(2 n+1) \sum_{k=0}^{n}(-1)^{k}\left(\left(\begin{array}{c}
2 n \\
n-k
\end{array}\right)-\left(\begin{array}{c}
2 n \\
n-k-1
\end{array}\right)\right)= \begin{cases}0, & \text { if } n>0, \\
1, & \text { if } n=0 .\end{cases}
\end{aligned}
$$

For $r \geq 1$, observing that

$$
\left(\begin{array}{c}
2 n+1 \\
n-k
\end{array}\right) k(k+1)=n(n+1)\left(\begin{array}{c}
2 n+1 \\
n-k
\end{array}\right)-2 n(2 n+1)\left(\begin{array}{c}
2 n-1 \\
n-k-1
\end{array}\right)
$$

we have

$$
\begin{aligned}
P_{r}(n) & =n(n+1) P_{r-1}(n)-2 n(2 n+1) P_{r-1}(n-1), \\
Q_{r}(n) & =n(n+1) Q_{r-1}(n)-2 n(2 n+1) Q_{r-1}(n-1)
\end{aligned}
$$

for $n \geq 1$. For the above recurrences we derive immediately that

$$
P_{1}(n)=n(2 n+1)\left(\begin{array}{c}
2 n \\
n
\end{array}\right), \quad P_{2}(n)=2 n^{2}(2 n+1)\left(\begin{array}{c}
2 n \\
n
\end{array}\right),
$$

and $Q_{1}(1)=-6, Q_{1}(2)=0, Q_{2}(1)=-12, Q_{2}(2)=120$, and $Q_{1}(n)=Q_{2}(n)=0$ for $n \geq 3$. Therefore, Theorem 2.1 is true for $r=0,1,2$. Now suppose that $r \geq 3$ and Theorem 2.1 holds for $r-1$. Then $P_{r-1}(n)$ is divisible by $(2 n+1)\left(\begin{array}{c}2 n \\ n\end{array}\right) n^{2}$, and $2 n(2 n+1) P_{r-1}(n-1)$ is divisible by

$$
2 n(2 n+1)(2 n-1)\left(\begin{array}{c}
2 n-2 \\
n-1
\end{array}\right)=(2 n+1)\left(\begin{array}{c}
2 n \\
n
\end{array}\right) n^{2} .
$$

By (2.1) we see that $P_{r}(n)$ is also divisible by $(2 n+1)\left(\begin{array}{c}2 n \\ n\end{array}\right) n^{2}$. This completes the inductive step for $P_{r}(n)$. Similarly, by $(2.1)$ we can prove the case for $Q_{r}(n)$.

We may also consider the following sums:

$$
\begin{aligned}
U_{r}(n) & :=\sum_{k=0}^{n}\left(\begin{array}{c}
2 n+1 \\
n-k
\end{array}\right)(2 k+1)^{2 r}, \\
V_{r}(n) & :=\sum_{k=0}^{n}(-1)^{k}\left(\begin{array}{c}
2 n+1 \\
n-k
\end{array}\right)(2 k+1)^{2 r} .
\end{aligned}
$$


It is easy to see that

$$
\begin{aligned}
\sum_{k=0}^{n}\left(\begin{array}{c}
2 n+1 \\
n-k
\end{array}\right) & =\frac{1}{2}\left(\sum_{k=0}^{n}\left(\begin{array}{c}
2 n+1 \\
k
\end{array}\right)+\sum_{k=0}^{n}\left(\begin{array}{c}
2 n+1 \\
n+1+k
\end{array}\right)\right)=\frac{1}{2}(1+1)^{2 n+1}=4^{n}, \\
\sum_{k=0}^{n}(-1)^{k}\left(\begin{array}{c}
2 n+1 \\
n-k
\end{array}\right) & =\sum_{k=0}^{n}(-1)^{k}\left(\left(\begin{array}{c}
2 n \\
n-k
\end{array}\right)+\left(\begin{array}{c}
2 n \\
n-k-1
\end{array}\right)\right)=\left(\begin{array}{c}
2 n \\
n
\end{array}\right) .
\end{aligned}
$$

Similarly to (2.1) and (2.2), we have

$$
\begin{aligned}
& U_{r}(n)=(2 n+1)^{2} U_{r-1}(n)-8 n(2 n+1) U_{r-1}(n-1), \\
& V_{r}(n)=(2 n+1)^{2} V_{r-1}(n)-8 n(2 n+1) V_{r-1}(n-1) .
\end{aligned}
$$

By (2.3)-(2.6) we immediately obtain the following result.

Corollary 2.2. For $n \in \mathbb{Z}^{+}$and $r \in \mathbb{N}$, there hold

$$
\begin{array}{r}
4^{-n} \sum_{k=0}^{n}\left(\begin{array}{c}
2 n+1 \\
n-k
\end{array}\right)(2 k+1)^{2 r} \equiv 1 \quad(\bmod 2), \\
2^{-\alpha(n)} \sum_{k=0}^{n}(-1)^{k}\left(\begin{array}{c}
2 n+1 \\
n-k
\end{array}\right)(2 k+1)^{2 r} \equiv 1 \quad(\bmod 2),
\end{array}
$$

where $\alpha(n)$ denotes the number of 1 's in the binary expansion of $n$.

\section{Proof of Theorem 1.2 for $m=2$}

We first give two combinatorial identities.

Lemma 3.1. For all $n_{1}, n_{2} \in \mathbb{N}$, there hold

$$
\begin{array}{r}
\sum_{k=0}^{n_{1}}\left(\begin{array}{c}
n_{1}+n_{2}+1 \\
n_{1}-k
\end{array}\right)\left(\begin{array}{c}
n_{1}+n_{2}+1 \\
n_{2}-k
\end{array}\right)(2 k+1)=\left(n_{1}+n_{2}+1\right)\left(\begin{array}{c}
n_{1}+n_{2} \\
n_{1}
\end{array}\right)^{2}, \\
\sum_{k=0}^{n_{1}}(-1)^{k}\left(\begin{array}{c}
n_{1}+n_{2}+1 \\
n_{1}-k
\end{array}\right)\left(\begin{array}{c}
n_{1}+n_{2}+1 \\
n_{2}-k
\end{array}\right)(2 k+1)=\left(n_{1}+n_{2}+1\right)\left(\begin{array}{c}
n_{1}+n_{2} \\
n_{1}
\end{array}\right) .
\end{array}
$$

Proof. It is easy to see that the left-hand side of (3.1) may be written as

$$
\begin{aligned}
& \sum_{k=0}^{n_{1}}\left(n_{2}+k+1\right)\left(\begin{array}{c}
n_{1}+n_{2}+1 \\
n_{1}-k
\end{array}\right)\left(\begin{array}{c}
n_{1}+n_{2} \\
n_{2}-k
\end{array}\right) \\
& \quad-\sum_{k=0}^{n_{1}}\left(n_{2}+k+2\right)\left(\begin{array}{c}
n_{1}+n_{2}+1 \\
n_{1}-k-1
\end{array}\right)\left(\begin{array}{c}
n_{1}+n_{2} \\
n_{2}-k-1
\end{array}\right) \\
& =\left(n_{2}+1\right)\left(\begin{array}{c}
n_{1}+n_{2}+1 \\
n_{1}
\end{array}\right)\left(\begin{array}{c}
n_{1}+n_{2} \\
n_{2}
\end{array}\right)
\end{aligned}
$$

which is equal to the right-hand side of (3.1).

Replacing $k$ by $-k-1$ in the left-hand side of $(3.2)$, we observe that

$$
\begin{aligned}
& \sum_{k=0}^{n_{1}}(-1)^{k}\left(\begin{array}{c}
n_{1}+n_{2}+1 \\
n_{1}-k
\end{array}\right)\left(\begin{array}{c}
n_{1}+n_{2}+1 \\
n_{2}-k
\end{array}\right)(2 k+1) \\
& \quad=\sum_{k=-n_{1}-1}^{-1}(-1)^{k}\left(\begin{array}{c}
n_{1}+n_{2}+1 \\
n_{1}-k
\end{array}\right)\left(\begin{array}{c}
n_{1}+n_{2}+1 \\
n_{2}-k
\end{array}\right)(2 k+1) .
\end{aligned}
$$


It follows that $(3.2)$ is equivalent to

$$
\sum_{k=-n_{1}-1}^{n_{1}}(-1)^{k}\left(\begin{array}{c}
n_{1}+n_{2}+1 \\
n_{1}-k
\end{array}\right)\left(\begin{array}{c}
n_{1}+n_{2}+1 \\
n_{2}-k
\end{array}\right)(2 k+1)=2\left(n_{1}+n_{2}+1\right)\left(\begin{array}{c}
n_{1}+n_{2} \\
n_{1}
\end{array}\right) .
$$

Since

$$
\begin{array}{r}
\left(\begin{array}{c}
n_{1}+n_{2}+1 \\
n_{1}-k
\end{array}\right)\left(\begin{array}{c}
n_{1}+n_{2}+1 \\
n_{2}-k
\end{array}\right)(2 k+1)=\left(n_{1}+n_{2}+1\right)\left(\begin{array}{c}
n_{1}+n_{2} \\
n_{1}-k
\end{array}\right)\left(\begin{array}{c}
n_{1}+n_{2}+1 \\
n_{2}-k
\end{array}\right) \\
-\left(n_{1}+n_{2}+1\right)\left(\begin{array}{c}
n_{1}+n_{2}+1 \\
n_{1}-k
\end{array}\right)\left(\begin{array}{c}
n_{1}+n_{2} \\
n_{2}-k-1
\end{array}\right)
\end{array}
$$

to prove (3.3), it suffices to establish the following two identities:

$$
\begin{array}{r}
\sum_{k=-n_{1}-1}^{n_{1}}(-1)^{k}\left(\begin{array}{c}
n_{1}+n_{2} \\
n_{1}-k
\end{array}\right)\left(\begin{array}{c}
n_{1}+n_{2}+1 \\
n_{2}-k
\end{array}\right)=\left(\begin{array}{c}
n_{1}+n_{2} \\
n_{1}
\end{array}\right), \\
\sum_{k=-n_{1}-1}^{n_{1}}(-1)^{k+1}\left(\begin{array}{c}
n_{1}+n_{2}+1 \\
n_{1}-k
\end{array}\right)\left(\begin{array}{c}
n_{1}+n_{2} \\
n_{2}-k-1
\end{array}\right)=\left(\begin{array}{c}
n_{1}+n_{2} \\
n_{1}
\end{array}\right),
\end{array}
$$

which follow immediately by comparing the coefficients of $x^{2 n_{2}}$ in the expansion of

$$
(1-x)^{n_{1}+n_{2}}(1+x)^{n_{1}+n_{2}+1}=(1+x)\left(1-x^{2}\right)^{n_{1}+n_{2}} .
$$

This completes the proof.

Remark. We can give another proof of (3.1) and (3.2) by computing their generating functions and using the following identity

$$
\sum_{m, n=0}^{\infty}\left(\begin{array}{c}
m+n+\alpha \\
m
\end{array}\right)\left(\begin{array}{c}
m+n+\beta \\
n
\end{array}\right) x^{m} y^{n}=\frac{2^{\alpha+\beta}}{\Delta(1-x+y+\Delta)^{\alpha}(1+x-y+\Delta)^{\beta}},
$$

where $\Delta:=\sqrt{1-2 x-2 y-2 x y+x^{2}+y^{2}}$. The identity (3.4) is equivalent to the generating function of Jacobi polynomials. See [1, p. 298], [13, p. 271] or [4] for a proof of this identity, and [9] for an application to prove some double-sum binomial coefficient identities.

As an example, we compute the generating function for the left-hand side of (3.2):

$$
\begin{aligned}
& \sum_{n_{1}, n_{2}=0}^{\infty} x^{n_{1}} y^{n_{2}} \sum_{k=0}^{\min \left\{n_{1}, n_{2}\right\}}(-1)^{k}\left(\begin{array}{c}
n_{1}+n_{2}+1 \\
n_{1}-k
\end{array}\right)\left(\begin{array}{c}
n_{1}+n_{2}+1 \\
n_{2}-k
\end{array}\right)(2 k+1) \\
& =\sum_{k=0}^{\infty}(2 k+1)(-x y)^{k} \sum_{n_{1}, n_{2}=k}^{\infty} x^{n_{1}-k} y^{n_{2}-k}\left(\begin{array}{c}
n_{1}+n_{2}+1 \\
n_{1}-k
\end{array}\right)\left(\begin{array}{c}
n_{1}+n_{2}+1 \\
n_{2}-k
\end{array}\right) \\
& =\sum_{k=0}^{\infty} \frac{2^{4 k+2}(2 k+1)(-x y)^{k}}{\Delta(1-x+y+\Delta)^{2 k+1}(1+x-y+\Delta)^{2 k+1}} \\
& =\sum_{k=0}^{\infty} \frac{2^{2 k+1}(2 k+1)(-x y)^{k}}{\Delta(1-x-y+\Delta)^{2 k+1}} \\
= & \frac{2\left(1-4 x y(1-x-y+\Delta)^{-2}\right)}{\Delta(1-x-y+\Delta)\left(1+4 x y(1-x-y+\Delta)^{-2}\right)^{2}} \\
= & \frac{1}{(1-x-y)^{2}} .
\end{aligned}
$$


Clearly the last expression is the generating function for the right-hand side of (3.2).

Let

$$
\begin{aligned}
P_{r}\left(n_{1}, n_{2}\right) & :=\sum_{k=0}^{n_{1}}\left(\begin{array}{c}
n_{1}+n_{2}+1 \\
n_{1}-k
\end{array}\right)\left(\begin{array}{c}
n_{1}+n_{2}+1 \\
n_{2}-k
\end{array}\right) k^{r}(k+1)^{r}(2 k+1), \\
Q_{r}\left(n_{1}, n_{2}\right) & :=\sum_{k=0}^{n_{1}}(-1)^{k}\left(\begin{array}{c}
n_{1}+n_{2}+1 \\
n_{1}-k
\end{array}\right)\left(\begin{array}{c}
n_{1}+n_{2}+1 \\
n_{2}-k
\end{array}\right) k^{r}(k+1)^{r}(2 k+1) .
\end{aligned}
$$

Then, the $m=2$ case of Theorem 1.2 may be stated as follows.

Theorem 3.2. For all $n_{1}, n_{2} \in \mathbb{Z}^{+}$and $r \in \mathbb{N}$, there hold

$$
\begin{gathered}
P_{r}\left(n_{1}, n_{2}\right) \equiv 0 \quad \bmod \left(n_{1}+n_{2}+1\right)\left(\begin{array}{c}
n_{1}+n_{2} \\
n_{1}
\end{array}\right) n_{1}^{\min \{1, r\}} n_{2}^{\min \left\{1,\left(\begin{array}{c}
r \\
2
\end{array}\right)\right\}}, \\
Q_{r}\left(n_{1}, n_{2}\right) \equiv 0 \quad \bmod \left(n_{1}+n_{2}+1\right)\left(\begin{array}{c}
n_{1}+n_{2} \\
n_{1}
\end{array}\right) n_{1}^{\min \{1, r\}} n_{2}^{\min \{1, r\}} .
\end{gathered}
$$

Proof of Theorem 3.2. We proceed by induction on $r$. Writing

$$
\begin{array}{r}
\left(\begin{array}{c}
n_{1}+n_{2}+1 \\
n_{1}-k
\end{array}\right)\left(\begin{array}{c}
n_{1}+n_{2}+1 \\
n_{2}-k
\end{array}\right) k(k+1)= \\
n_{1}\left(n_{1}+1\right)\left(\begin{array}{c}
n_{1}+n_{2}+1 \\
n_{1}-k
\end{array}\right)\left(\begin{array}{c}
n_{1}+n_{2}+1 \\
n_{2}-k
\end{array}\right) \\
-\left(n_{1}+n_{2}+1\right)^{2}\left(\begin{array}{c}
n_{1}+n_{2} \\
n_{1}-k-1
\end{array}\right)\left(\begin{array}{c}
n_{1}+n_{2} \\
n_{2}-k
\end{array}\right),
\end{array}
$$

we derive

$$
\begin{aligned}
& P_{r}\left(n_{1}, n_{2}\right)=n_{1}\left(n_{1}+1\right) P_{r-1}\left(n_{1}, n_{2}\right)-\left(n_{1}+n_{2}+1\right)^{2} P_{r-1}\left(n_{1}-1, n_{2}\right), \\
& Q_{r}\left(n_{1}, n_{2}\right)=n_{1}\left(n_{1}+1\right) Q_{r-1}\left(n_{1}, n_{2}\right)-\left(n_{1}+n_{2}+1\right)^{2} Q_{r-1}\left(n_{1}-1, n_{2}\right), \quad r \geq 1 .
\end{aligned}
$$

From the above recurrences and Lemma 3.1 we immediately get

$$
\begin{aligned}
& P_{1}\left(n_{1}, n_{2}\right)=n_{1}\left(n_{1}+n_{2}+1\right)\left(\begin{array}{c}
n_{1}+n_{2} \\
n_{1}
\end{array}\right)\left(\begin{array}{c}
n_{1}+n_{2}-1 \\
n_{1}
\end{array}\right), \\
& P_{2}\left(n_{1}, n_{2}\right)=2 n_{1} n_{2}\left(n_{1}+n_{2}+1\right)\left(\begin{array}{c}
n_{1}+n_{2} \\
n_{1}
\end{array}\right)\left(\begin{array}{c}
n_{1}+n_{2}-2 \\
n_{1}-1
\end{array}\right), \\
& Q_{1}\left(n_{1}, n_{2}\right)=-n_{1} n_{2}\left(n_{1}+n_{2}+1\right)\left(\begin{array}{c}
n_{1}+n_{2} \\
n_{1}
\end{array}\right), \\
& Q_{2}\left(n_{1}, n_{2}\right)=n_{1} n_{2}\left(n_{1} n_{2}-n_{1}-n_{1}-1\right)\left(n_{1}+n_{2}+1\right)\left(\begin{array}{c}
n_{1}+n_{2} \\
n_{1}
\end{array}\right) .
\end{aligned}
$$

Therefore, Theorem 3.2 is true for $r=0,1,2$. Now suppose that the statement is true for $r-1(r \geq 3)$. Then $n_{1} P_{r-1}\left(n_{1}, n_{2}\right)$ is divisible by $n_{1} n_{2}\left(n_{1}+n_{2}+1\right)\left(\begin{array}{c}n_{1}+n_{2} \\ n_{1}\end{array}\right)$ and $\left(n_{1}+n_{2}+1\right) P_{r-1}\left(n_{1}-1, n_{2}\right)$ is divisible by

$$
\left(n_{1}+n_{2}+1\right) n_{2}\left(n_{1}+n_{2}\right)\left(\begin{array}{c}
n_{1}+n_{2}-1 \\
n_{1}-1
\end{array}\right)=n_{1} n_{2}\left(n_{1}+n_{2}+1\right)\left(\begin{array}{c}
n_{1}+n_{2} \\
n_{1}
\end{array}\right) .
$$

By (3.5), we see that $P_{r}\left(n_{1}, n_{2}\right)$ is divisible by $n_{1} n_{2}\left(n_{1}+n_{2}+1\right)\left(\begin{array}{c}n_{1}+n_{2} \\ n_{1}\end{array}\right)$, and we complete the inductive step for $P_{r}\left(n_{1}, n_{2}\right)$. The case for $Q_{r}\left(n_{1}, n_{2}\right)$ is exactly the same.

Remark. Similarly, if we set

$$
\bar{P}_{r}\left(n_{1}, n_{2}\right):=\sum_{k=0}^{n_{1}}\left(\begin{array}{c}
n_{1}+n_{2} \\
n_{1}-k
\end{array}\right)\left(\begin{array}{c}
n_{1}+n_{2} \\
n_{2}-k
\end{array}\right) k^{2 r+1}
$$


then

$$
\begin{aligned}
& \bar{P}_{0}\left(n_{1}, n_{2}\right)=\frac{n_{1}}{2}\left(\begin{array}{c}
n_{1}+n_{2} \\
n_{1}
\end{array}\right)\left(\begin{array}{c}
n_{1}+n_{2}-1 \\
n_{1}
\end{array}\right), \\
& \bar{P}_{r}\left(n_{1}, n_{2}\right)=n_{1}^{2} \bar{P}_{r-1}\left(n_{1}, n_{2}\right)-\left(n_{1}+n_{2}\right)^{2} \bar{P}_{r-1}\left(n_{1}-1, n_{2}\right), \quad r \geq 1,
\end{aligned}
$$

from which we can deduce that $2 \bar{P}_{r}\left(n_{1}, n_{2}\right)$ is divisible by $n_{1}\left(\begin{array}{c}n_{1}+n_{2} \\ n_{1}\end{array}\right)$ by induction on $r$. This result is the base of the inductive proof of [10, Theorem 1.3], though it was not explicitly stated there.

\section{Proof of Theorem 1.2 for $m \geq 3$}

For all nonnegative integers $a_{1}, \ldots, a_{l}$, and $k$, let

$$
C\left(a_{1}, \ldots, a_{l} ; k\right)=\prod_{i=1}^{l}\left(\begin{array}{c}
a_{i}+a_{i+1}+1 \\
a_{i}-k
\end{array}\right),
$$

where $a_{l+1}=a_{1}$, and let

$$
\begin{aligned}
& S_{r}\left(n_{1}, \ldots, n_{m}\right)=\frac{n_{1} ! n_{m} !}{\left(n_{1}+n_{m}+1\right) !} \sum_{k=0}^{n_{1}} k^{r}(k+1)^{r}(2 k+1) C\left(n_{1}, \ldots, n_{m} ; k\right), \\
& T_{r}\left(n_{1}, \ldots, n_{m}\right)=\frac{n_{1} ! n_{m} !}{\left(n_{1}+n_{m}+1\right) !} \sum_{k=0}^{n_{1}}(-1)^{k} k^{r}(k+1)^{r}(2 k+1) C\left(n_{1}, \ldots, n_{m} ; k\right) .
\end{aligned}
$$

Observe that, for $m \geq 3$, we have

$$
\begin{aligned}
& C\left(n_{1}, \ldots, n_{m} ; k\right) \\
& \quad=\frac{\left(n_{2}+n_{3}+1\right) !\left(n_{m}+n_{1}+1\right) !}{\left(n_{1}+k+1\right) !\left(n_{2}-k\right) !\left(n_{m}+n_{3}+1\right) !}\left(\begin{array}{c}
n_{1}+n_{2}+1 \\
n_{1}-k
\end{array}\right) C\left(n_{3}, \ldots, n_{m} ; k\right),
\end{aligned}
$$

and by the Chu-Vandermonde formula (see $[1$, p. 67]) we have

$$
\left(\begin{array}{c}
n_{1}+n_{2}+1 \\
n_{1}-k
\end{array}\right)=\sum_{s=0}^{n_{1}-k} \frac{\left(n_{1}+k+1\right) !\left(n_{2}-k\right) !}{s !(s+2 k+1) !\left(n_{1}-k-s\right) !\left(n_{2}-k-s\right) !} .
$$

Substituting (4.3) and (4.4) into the right-hand side of (4.1), we get

$$
\begin{aligned}
S_{r}\left(n_{1}, \ldots, n_{m}\right) & =\frac{\left(n_{2}+n_{3}+1\right) ! n_{1} ! n_{m} !}{\left(n_{m}+n_{3}+1\right) !} \sum_{k=0}^{n_{1}} \sum_{s=0}^{n_{1}-k} \frac{(2 k+1)^{2 r+1} C\left(n_{3}, \ldots, n_{m} ; k\right)}{s !(s+2 k+1) !\left(n_{1}-k-s\right) !\left(n_{2}-k-s\right) !} \\
& =\frac{\left(n_{2}+n_{3}+1\right) ! n_{1} ! n_{m} !}{\left(n_{m}+n_{3}+1\right) !} \sum_{l=0}^{n_{1}} \sum_{k=0}^{l} \frac{(2 k+1)^{2 r+1} C\left(n_{3}, \ldots, n_{m} ; k\right)}{(l-k) !(l+k+1) !\left(n_{1}-l\right) !\left(n_{2}-l\right) !},
\end{aligned}
$$

where $l=s+k$. Now, in the last sum making the substitution

$$
\frac{C\left(n_{3}, \ldots, n_{m} ; k\right)}{(l-k) !(l+k+1) !}=\frac{\left(n_{m}+n_{3}+1\right) !}{\left(n_{3}+l+1\right) !\left(n_{m}+l+1\right) !} C\left(l, n_{3}, \ldots, n_{m} ; k\right),
$$

we obtain the following recurrence relation

$$
S_{r}\left(n_{1}, \ldots, n_{m}\right)=\sum_{l=0}^{n_{1}}\left(\begin{array}{c}
n_{1} \\
l
\end{array}\right)\left(\begin{array}{c}
n_{2}+n_{3}+1 \\
n_{2}-l
\end{array}\right) S_{r}\left(l, n_{3}, \ldots, n_{m}\right), \quad m \geq 3 .
$$


Similarly, we have

$$
T_{r}\left(n_{1}, \ldots, n_{m}\right)=\sum_{l=0}^{n_{1}}\left(\begin{array}{c}
n_{1} \\
l
\end{array}\right)\left(\begin{array}{c}
n_{2}+n_{3}+1 \\
n_{2}-l
\end{array}\right) T_{r}\left(l, n_{3}, \ldots, n_{m}\right), \quad m \geq 3 .
$$

We now proceed by induction on $m$. By Theorem 3.2, we suppose that Theorem 1.2 is true for $m-1$ $(m \geq 3)$. If $r=0$, then by (4.5) and (4.6), Theorem 1.2 is true for $m$. If $r \geq 1$, then by definition,

$$
S_{r}\left(0, n_{3}, \ldots, n_{m}\right)=T_{r}\left(0, n_{3}, \ldots, n_{m}\right)=0,
$$

and by the induction hypothesis,

$$
\begin{array}{ll}
S_{r}\left(l, n_{3}, \ldots, n_{m}\right) \equiv 0 & \bmod \ell^{\min \{1, r\}} n_{m}^{\min \left\{1,\left(\begin{array}{c}
r \\
2
\end{array}\right)\right\}}, \\
T_{r}\left(l, n_{3}, \ldots, n_{m}\right) \equiv 0 & \bmod \ell^{\min \{1, r\}} n_{m}^{\min \{1, r\}}
\end{array}
$$

for $\ell \geq 1$. Hence, by (4.5) and (4.6) and noticing that $\left(\begin{array}{c}n_{1} \\ \ell\end{array}\right) \ell=n_{1}\left(\begin{array}{c}n_{1}-1 \\ \ell-1\end{array}\right)$, we get

$$
\begin{array}{ll}
S_{r}\left(n_{1}, \ldots, n_{m}\right) \equiv 0 & \bmod n_{1}^{\min \{1, r\}} n_{m}^{\min \left\{1,\left(\begin{array}{c}
r \\
2
\end{array}\right)\right\}}, \\
T_{r}\left(n_{1}, \ldots, n_{m}\right) \equiv 0 & \bmod n_{1}^{\min \{1, r\}} n_{m}^{\min \{1, r\}},
\end{array}
$$

Namely, Theorem 1.2 is true for $m \geq 3$. This completes the proof.

By repeatedly using (4.5) and (4.6) for $r=0,1$, we obtain the following results.

Corollary 4.1. For all $m \geq 3$ and $n_{1}, \ldots, n_{m} \in \mathbb{Z}^{+}$, there hold

$$
\begin{aligned}
& \sum_{k=0}^{n_{1}}(2 k+1) \prod_{i=1}^{m}\left(\begin{array}{c}
n_{i}+n_{i+1}+1 \\
n_{i}-k
\end{array}\right) \\
& \quad=\left(n_{1}+n_{m}+1\right)\left(\begin{array}{c}
n_{1}+n_{m} \\
n_{1}
\end{array}\right) \sum_{\lambda}\left(\begin{array}{c}
\lambda_{m-2}+n_{m} \\
\lambda_{m-2}
\end{array}\right) \prod_{i=1}^{m-2}\left(\begin{array}{c}
\lambda_{i-1} \\
\lambda_{i}
\end{array}\right)\left(\begin{array}{c}
n_{i+1}+n_{i+2}+1 \\
n_{i+1}-\lambda_{i}
\end{array}\right), \\
& \sum_{k=0}^{n_{1}} k(k+1)(2 k+1) \prod_{i=1}^{m}\left(\begin{array}{c}
n_{i}+n_{i+1}+1 \\
n_{i}-k
\end{array}\right) \\
& \quad=n_{m}\left(n_{1}+n_{m}+1\right)\left(\begin{array}{c}
n_{1}+n_{m} \\
n_{1}
\end{array}\right) \sum_{\lambda}\left(\begin{array}{c}
\lambda_{m-2}+n_{m}-1 \\
\lambda_{m-2}-1
\end{array}\right) \prod_{i=1}^{m-2}\left(\begin{array}{c}
\lambda_{i-1} \\
\lambda_{i}
\end{array}\right)\left(\begin{array}{c}
n_{i+1}+n_{i+2}+1 \\
n_{i+1}-\lambda_{i}
\end{array}\right),
\end{aligned}
$$

where $n_{m+1}=\lambda_{0}=n_{1}$ and the sums are over all sequences $\lambda=\left(\lambda_{1}, \ldots, \lambda_{m-2}\right)$ of nonnegative integers such that $n_{1} \geq \lambda_{1} \geq \cdots \geq \lambda_{m-2}$.

Corollary 4.2. For all $m \geq 3$ and $n_{1}, \ldots, n_{m} \in \mathbb{Z}^{+}$, there hold

$$
\begin{aligned}
& \sum_{k=0}^{n_{1}}(-1)^{k}(2 k+1) \prod_{i=1}^{m}\left(\begin{array}{c}
n_{i}+n_{i+1}+1 \\
n_{i}-k
\end{array}\right) \\
& \quad=\left(n_{1}+n_{m}+1\right)\left(\begin{array}{c}
n_{1}+n_{m} \\
n_{1}
\end{array}\right) \sum_{\lambda} \prod_{i=1}^{m-2}\left(\begin{array}{c}
\lambda_{i-1} \\
\lambda_{i}
\end{array}\right)\left(\begin{array}{c}
n_{i+1}+n_{i+2}+1 \\
n_{i+1}-\lambda_{i}
\end{array}\right), \\
& \sum_{k=0}^{n_{1}}(-1)^{k+1} k(k+1)(2 k+1) \prod_{i=1}^{m}\left(\begin{array}{c}
n_{i}+n_{i+1}+1 \\
n_{i}-k
\end{array}\right) \\
& \quad=n_{m}\left(n_{1}+n_{m}+1\right)\left(\begin{array}{c}
n_{1}+n_{m} \\
n_{1}
\end{array}\right) \sum_{\lambda} \lambda_{m-2} \prod_{i=1}^{m-2}\left(\begin{array}{c}
\lambda_{i-1} \\
\lambda_{i}
\end{array}\right)\left(\begin{array}{c}
n_{i+1}+n_{i+2}+1 \\
n_{i+1}-\lambda_{i}
\end{array}\right),
\end{aligned}
$$

where $n_{m+1}=\lambda_{0}=n_{1}$ and the sums are over all sequences $\lambda=\left(\lambda_{1}, \ldots, \lambda_{m-2}\right)$ of nonnegative integers such that $n_{1} \geq \lambda_{1} \geq \cdots \geq \lambda_{m-2}$. 
Note that the above identities show that the alternating sums in the left-hand sides are positive. When $m=3$, applying the Chu-Vandermonde formula, we derive the following identities from Corollary 4.2:

$$
\begin{aligned}
& \sum_{k=0}^{n_{1}}(-1)^{k}(2 k+1)\left(\begin{array}{c}
n_{1}+n_{2}+1 \\
n_{1}-k
\end{array}\right)\left(\begin{array}{c}
n_{2}+n_{3}+1 \\
n_{2}-k
\end{array}\right)\left(\begin{array}{c}
n_{3}+n_{1}+1 \\
n_{3}-k
\end{array}\right)=\frac{\left(n_{1}+n_{2}+n_{3}+1\right) !}{n_{1} ! n_{2} ! n_{3} !} \\
& \sum_{k=0}^{n_{1}}(-1)^{k+1} k(k+1)(2 k+1)\left(\begin{array}{c}
n_{1}+n_{2}+1 \\
n_{1}-k
\end{array}\right)\left(\begin{array}{c}
n_{2}+n_{3}+1 \\
n_{2}-k
\end{array}\right)\left(\begin{array}{c}
n_{3}+n_{1}+1 \\
n_{3}-k
\end{array}\right)=\frac{\left(n_{1}+n_{2}+n_{3}\right) !}{\left(n_{1}-1\right) !\left(n_{2}-1\right) !\left(n_{3}-1\right) !}
\end{aligned}
$$

\section{Proof of Theorem 1.4}

In this section we shall use the following theorem of Lucas (see, for example, [7]). We refer the reader to $[10,14,16]$ for recent applications of Lucas' theorem.

Lemma 5.1 (Lucas' theorem). Let $p$ be a prime, and let $a_{0}, b_{0}, \ldots, a_{m}, b_{m} \in\{0, \ldots, p-1\}$. Then

$$
\left(\begin{array}{c}
a_{0}+a_{1} p+\cdots+a_{m} p^{m} \\
b_{0}+b_{1} p+\cdots+b_{m} p^{m}
\end{array}\right) \equiv \prod_{i=0}^{m}\left(\begin{array}{l}
a_{i} \\
b_{i}
\end{array}\right) \quad(\bmod p) .
$$

Suppose that $r+s \equiv 1(\bmod 2)$ and $s \geq 1$. Putting $m=s$ and $n_{1}=\cdots=n_{s}=n$ in Theorem 1.1, we see that

$$
\sum_{k=0}^{n}(2 k+1)^{r+s}\left(\begin{array}{c}
2 n+1 \\
n-k
\end{array}\right)^{s} \equiv \sum_{k=0}^{n}(-1)^{k}(2 k+1)^{r+s}\left(\begin{array}{c}
2 n+1 \\
n-k
\end{array}\right)^{s} \equiv 0 \bmod (2 n+1)\left(\begin{array}{c}
2 n \\
n
\end{array}\right) .
$$

Therefore, by the definition of $A_{n, k}$, we have

$$
\sum_{k=0}^{n}(2 k+1)^{r} A_{n, k}^{s} \equiv \sum_{k=0}^{n}(2 k+1)^{r}(-1)^{k} A_{n, k}^{s} \equiv 0 \bmod \frac{\left(\begin{array}{c}
2 n \\
n
\end{array}\right)}{\operatorname{gcd}\left(\left(\begin{array}{c}
2 n \\
n
\end{array}\right),(2 n+1)^{s-1}\right)},
$$

and so the congruence (1.1) holds if $s=1$.

Now suppose that $2 n+1=p^{a}(p \geq 3)$ is a prime power. By Lucas' theorem, we have

$$
\left(\begin{array}{c}
2 n \\
n
\end{array}\right)=\left(\begin{array}{c}
p^{a}-1 \\
\left(p^{a}-1\right) / 2
\end{array}\right) \equiv\left(\begin{array}{c}
p-1 \\
(p-1) / 2
\end{array}\right)^{a} \equiv(-1)^{a(p-1) / 2} \quad(\bmod p),
$$

which means that

$$
\operatorname{gcd}\left(\left(\begin{array}{c}
2 n \\
n
\end{array}\right),(2 n+1)^{s}\right)=1 .
$$

This completes the proof of Theorem 1.4.

Remark. It is natural to wonder which numbers $n$ satisfy (5.1) besides those we just mentioned. Via MAPLE, we find that all such numbers $n$ less than 300 are as follows:

$$
\begin{aligned}
& 10,27,28,32,37,57,59,66,85,91,101,108,109,118,126,132,137,150,152,159, \\
& 164,170,177,182,188,201,240,244,252,253,257,258,271,274,282,291 .
\end{aligned}
$$

That is to say, Theorem 1.4 is also true for these numbers $n$. On the other hand, it is easy to see from Lucas' theorem that, if $p$ and $p^{2}-p+1$ are both odd primes, then $n=\left(p^{3}-p^{2}+p-1\right) / 2$ satisfies (5.1). Via MAPLE, we find that there are 5912 such primes $p$ among the first $10^{5}$ primes. Here we list the first 20 such primes:

$$
3,7,13,67,79,139,151,163,193,337,349,379,457,541,613,643,727,769,919,991 .
$$




\section{Consequences of Theorem 1.1 and open problems}

For convenience, let $\varepsilon= \pm 1$ throughout this section. We shall give several interesting consequences of

Theorem 1.1 in this section. Note that we can also give the corresponding consequences of Theorem 1.2 in the same way.

Letting $n_{1}=\cdots=n_{m}=n$ in Theorem 1.1, we have

Corollary 6.1. For all $m, n \in \mathbb{Z}^{+}$and $r \in \mathbb{N}$, there holds

$$
\sum_{k=0}^{n} \varepsilon^{k}(2 k+1)^{2 r+1}\left(\begin{array}{c}
2 n+1 \\
n-k
\end{array}\right)^{m} \equiv 0 \bmod (2 n+1)\left(\begin{array}{c}
2 n \\
n
\end{array}\right)
$$

For $m=2 a+1 \geq 3$, we propose the following conjecture:

Conjecture 6.2. For all $a, n \in \mathbb{Z}^{+}$and $r \in \mathbb{N}$, there hold

$$
\begin{aligned}
&\left(\begin{array}{c}
2 n \\
n
\end{array}\right)^{-1} \sum_{k=0}^{n}(2 k+1)^{2 r+1}\left(\begin{array}{c}
2 n+1 \\
n-k
\end{array}\right)^{2 a+1} \equiv\left\{\begin{array}{ll}
1, & \text { if } n=2^{b}-1 \\
0, & \text { otherwise }
\end{array} \quad \bmod 2,\right. \\
&\left(\begin{array}{c}
2 n \\
n
\end{array}\right)^{-1} \sum_{k=0}^{n}(-1)^{k}(2 k+1)^{2 r+1}\left(\begin{array}{c}
2 n+1 \\
n-k
\end{array}\right)^{2 a+1} \equiv\left\{\begin{array}{ll}
1, & \text { if } n=2^{b}+2^{c}(b, c \in \mathbb{N}) \\
0, & \text { otherwise }
\end{array} \quad \bmod 2 .\right.
\end{aligned}
$$

Letting $n_{2 i-1}=m$ and $n_{2 i}=n$ for $1 \leq i \leq a$ in Theorem 1.1, we obtain

Corollary 6.3. For all $a, m, n \in \mathbb{Z}^{+}$and $r \in \mathbb{N}$, there holds

$$
\sum_{k=0}^{m} \varepsilon^{k}(2 k+1)^{2 r+1}\left(\begin{array}{c}
m+n+1 \\
m-k
\end{array}\right)^{a}\left(\begin{array}{c}
m+n+1 \\
n-k
\end{array}\right)^{a} \equiv 0 \bmod (m+n+1)\left(\begin{array}{c}
m+n \\
m
\end{array}\right) .
$$

Letting $n_{3 i-2}=l, n_{3 i-1}=m$ and $n_{3 i}=n$ for $1 \leq i \leq a$ in Theorem 1.1, we obtain

Corollary 6.4. For all $a, l, m, n \in \mathbb{Z}^{+}$and $r \in \mathbb{N}$, there holds

$$
\sum_{k=0}^{l} \varepsilon^{k}(2 k+1)^{2 r+1}\left(\begin{array}{c}
l+m+1 \\
l-k
\end{array}\right)^{a}\left(\begin{array}{c}
m+n+1 \\
m-k
\end{array}\right)^{a}\left(\begin{array}{c}
n+l+1 \\
n-k
\end{array}\right)^{a} \equiv 0 \bmod (l+m+1)\left(\begin{array}{c}
l+m \\
l
\end{array}\right) .
$$

Letting $m=2 a+b, n_{1}=n_{3}=\cdots=n_{2 a-1}=n$ and letting all the other $n_{i}$ be $n-1$ in Theorem 1.1, we get

Corollary 6.5. For all $a, n \in \mathbb{Z}^{+}$and $b, r \in \mathbb{N}$, there holds

$$
\sum_{k=0}^{n} \varepsilon^{k}(2 k+1)^{2 r+1}\left(\begin{array}{c}
2 n \\
n-k
\end{array}\right)^{a}\left(\begin{array}{c}
2 n \\
n-k-1
\end{array}\right)^{a}\left(\begin{array}{c}
2 n-1 \\
n-k-1
\end{array}\right)^{b} \equiv 0 \bmod n\left(\begin{array}{c}
2 n \\
n
\end{array}\right)
$$

It is easy to see that Theorem 1.1 can be restated in the following form.

Theorem 6.6. For all $n_{1}, \ldots, n_{m} \in \mathbb{Z}^{+}$and $r \in \mathbb{N}$, the expression

$$
n_{1} ! \prod_{i=1}^{m} \frac{\left(n_{i}+n_{i+1}+1\right) !}{\left(2 n_{i}+1\right) !} \sum_{k=0}^{n_{1}} \varepsilon^{k}(2 k+1)^{2 r+1} \prod_{i=1}^{m}\left(\begin{array}{c}
2 n_{i}+1 \\
n_{i}-k
\end{array}\right) \quad\left(n_{m+1}=-1\right)
$$

is always an integer. 
It is easy to see (cf. [15]) that, for all $m, n \in \mathbb{N}$, the numbers $\frac{(2 m+1) !(2 n+1) !}{(m+n+1) ! m ! n !}$ and $\frac{(2 m) !(2 n) !}{(m+n) ! m ! n !}$ are integers by considering the $p$-adic order of a factorial. Letting $n_{1}=\cdots=n_{a}=m$ and $n_{a+1}=\cdots=n_{a+b}=n$ in Theorem 6.6, we obtain

Corollary 6.7. For all $a, b, m, n \in \mathbb{Z}^{+}$and $r \in \mathbb{N}$, there holds

$$
\sum_{k=0}^{m} \varepsilon^{k}(2 k+1)^{2 r+1}\left(\begin{array}{c}
2 m+1 \\
m-k
\end{array}\right)^{a}\left(\begin{array}{c}
2 n+1 \\
n-k
\end{array}\right)^{b} \equiv 0 \bmod \frac{(2 m+1) !(2 n+1) !}{(m+n+1) ! m ! n !}
$$

For example, we have

$$
\begin{gathered}
\sum_{k=0}^{n} \varepsilon^{k}(2 k+1)^{2 r+1}\left(\begin{array}{c}
2 n+3 \\
n-k+1
\end{array}\right)^{a}\left(\begin{array}{c}
2 n+1 \\
n-k
\end{array}\right)^{b} \equiv 0 \bmod (2 n+3)\left(\begin{array}{c}
2 n+1 \\
n
\end{array}\right), \\
\sum_{k=0}^{n} \varepsilon^{k}(2 k+1)^{2 r+1}\left(\begin{array}{c}
4 n+1 \\
2 n-k
\end{array}\right)^{a}\left(\begin{array}{c}
2 n+1 \\
n-k
\end{array}\right)^{b} \equiv 0 \bmod (2 n+1)\left(\begin{array}{c}
4 n+1 \\
n
\end{array}\right), \\
\sum_{k=0}^{n} \varepsilon^{k}(2 k+1)^{2 r+1}\left(\begin{array}{c}
6 n+1 \\
3 n-k
\end{array}\right)^{a}\left(\begin{array}{c}
2 n+1 \\
n-k
\end{array}\right)^{b} \equiv 0 \bmod \frac{(6 n+1) !(2 n+1) !}{(4 n+1) !(3 n) ! n !}
\end{gathered}
$$

Similarly to the proof of Theorem 1.4, we can deduce the following results.

Corollary 6.8. Let $r \in \mathbb{N}$ and $s, t \in \mathbb{Z}^{+}$such that $r+s+t \equiv 1(\bmod 2)$, and let $n \in \mathbb{Z}^{+}$. If $s=t=1$ or $\operatorname{gcd}\left(\frac{1}{2 n+1}\left(\begin{array}{c}2 n+1 \\ n\end{array}\right),(2 n+1)(2 n+3)\right)=1$, then

$$
\sum_{k=0}^{n-1} \varepsilon^{k}(2 k+1)^{r} A_{n+1, k}^{s} A_{n, k}^{t} \equiv 0 \quad \bmod \frac{1}{2 n+1}\left(\begin{array}{c}
2 n+1 \\
n
\end{array}\right) .
$$

In particular, if $2 n+1$ and $2 n+3$ are both prime powers, then (6.2) holds.

Corollary 6.9. Let $r \in \mathbb{N}$ and $s, t \in \mathbb{Z}^{+}$such that $r+s+t \equiv 1(\bmod 2)$, and let $n \in \mathbb{Z}^{+}$. If $s=t=1$ or $\operatorname{gcd}\left(\frac{1}{4 n+1}\left(\begin{array}{c}4 n+1 \\ n\end{array}\right),(2 n+1)(4 n+1)\right)=1$, then

$$
\sum_{k=0}^{n} \varepsilon^{k}(2 k+1)^{r} A_{2 n, k}^{s} A_{n, k}^{t} \equiv 0 \quad \bmod \frac{1}{4 n+1}\left(\begin{array}{c}
4 n+1 \\
n
\end{array}\right) .
$$

In particular, if $2 n+1$ and $4 n+1$ are both prime powers, then (6.3) holds.

Conjecture 6.10. The congruences (6.2) and (6.3) hold for all $n \in \mathbb{Z}^{+}$and $r, s, t$ given in Corollary 6.8 .

Conjecture 6.11. There are infinitely many numbers $n \in \mathbb{Z}^{+}$such that

$$
\operatorname{gcd}\left(\frac{1}{2 n+1}\left(\begin{array}{c}
2 n+1 \\
n
\end{array}\right),(2 n+1)(2 n+3)\right)=1 .
$$

Conjecture 6.12. There are infinitely many numbers $n \in \mathbb{Z}^{+}$such that

$$
\operatorname{gcd}\left(\frac{1}{4 n+1}\left(\begin{array}{c}
4 n+1 \\
n
\end{array}\right),(2 n+1)(4 n+1)\right)=1 .
$$




\begin{tabular}{|c|c|c|c|c|c|c|c|c|c|c|c|c|}
\hline$n$ & 1 & 10 & 20 & 50 & 100 & 200 & 500 & 1000 & 2000 & 3000 & 4000 & 5000 \\
\hline$f(n)$ & 1 & 8 & 13 & 24 & 38 & 59 & 104 & 167 & 255 & 353 & 439 & 508 \\
\hline$g(n)$ & 1 & 7 & 14 & 23 & 37 & 56 & 108 & 169 & 270 & 366 & 445 & 523 \\
\hline
\end{tabular}

Table 1: Some values of $f(x)$ and $g(x)$.

Let $f(x)$ and $g(x)$ denote the numbers of positive integers $n \leq x$ satisfying (6.4) and (6.5) respectively. Via Maple, we find that $f(x)$ and $g(x)$ grow a little slowly with respect to $x$. Table 1 gives some values of $f(x)$ and $g(x)$.

Using Lucas' theorem, it is easy to see that if $2 n+1$ and $2 n+3$ are both prime powers, then (6.4) holds. It is well known that the twin prime conjecture states that there are infinitely many primes $p$ such that $p+2$ is also prime. Therefore, if the twin prime conjecture is true, then so is Conjecture 6.11. Since the twin prime conjecture is rather difficult, we hope that Conjecture 6.11 might be tackled in another way.

In fact, we have the following more general conjecture:

Conjecture 6.13. For all $r \in \mathbb{N}$ and $m, n, s, t \in \mathbb{Z}^{+}$such that $r+s+t \equiv 1(\bmod 2)$, there holds

$$
(m+n+1) \sum_{k=0}^{m} \varepsilon^{k}(2 k+1)^{r} A_{m, k}^{s} A_{n, k}^{t} \equiv 0 \quad \bmod \frac{(2 m) !(2 n) !}{(m+n) ! m ! n !} .
$$

Note that the numbers $\frac{(2 m) !(2 n) !}{(m+n) ! m ! n !}$ are called super Catalan numbers, of which no combinatorial interpretations are known for general $m$ and $n$ until now. See Gessel [6] and Georgiadis et al. [5].

From Theorem 6.6 it is easy to see that, for all $a_{1}, \ldots, a_{m} \in \mathbb{Z}^{+}$,

$$
n_{1} ! \prod_{i=1}^{m} \frac{\left(n_{i}+n_{i+1}+1\right) !}{\left(2 n_{i}+1\right) !} \sum_{k=0}^{n_{1}} \varepsilon^{k}(2 k+1)^{2 r+1} \prod_{i=1}^{m}\left(\begin{array}{c}
2 n_{i}+1 \\
n_{i}-k
\end{array}\right)^{a_{i}} \quad\left(n_{m+1}=-1\right)
$$

is an integer. For $m=3$, letting $\left(n_{1}, n_{2}, n_{3}\right)$ be $(n, n+2, n+1),(n, 3 n, 2 n),(2 n, n, 3 n),(2 n, n, 4 n)$, or $(3 n, 2 n, 4 n)$, we obtain the following three corollaries.

Corollary 6.14. For all $a, b, c, n \in \mathbb{Z}^{+}$and $r \in \mathbb{N}$, there holds

$$
\sum_{k=0}^{n} \varepsilon^{k}(2 k+1)^{2 r+1}\left(\begin{array}{c}
2 n+1 \\
n-k
\end{array}\right)^{a}\left(\begin{array}{c}
2 n+3 \\
n-k+1
\end{array}\right)^{b}\left(\begin{array}{c}
2 n+5 \\
n-k+2
\end{array}\right)^{c} \equiv 0 \bmod (2 n+5)\left(\begin{array}{c}
2 n+1 \\
n
\end{array}\right) .
$$

Corollary 6.15. For all $a, b, c, n \in \mathbb{Z}^{+}$and $r \in \mathbb{N}$, there hold

$$
\begin{aligned}
& \sum_{k=0}^{n} \varepsilon^{k}(2 k+1)^{2 r+1}\left(\begin{array}{c}
6 n+1 \\
3 n-k
\end{array}\right)^{a}\left(\begin{array}{c}
4 n+1 \\
2 n-k
\end{array}\right)^{b}\left(\begin{array}{c}
2 n+1 \\
n-k
\end{array}\right)^{c} \equiv 0 \bmod (2 n+1)\left(\begin{array}{c}
6 n+1 \\
n
\end{array}\right), \\
& \sum_{k=0}^{n} \varepsilon^{k}(2 k+1)^{2 r+1}\left(\begin{array}{c}
6 n+1 \\
3 n-k
\end{array}\right)^{a}\left(\begin{array}{c}
4 n+1 \\
2 n-k
\end{array}\right)^{b}\left(\begin{array}{c}
2 n+1 \\
n-k
\end{array}\right)^{c} \equiv 0 \bmod (2 n+1)\left(\begin{array}{c}
6 n+1 \\
3 n
\end{array}\right) .
\end{aligned}
$$

Corollary 6.16. For all $a, b, c, n \in \mathbb{Z}^{+}$and $r \in \mathbb{N}$, there hold

$$
\begin{aligned}
& 2 \sum_{k=0}^{n} \varepsilon^{k}(2 k+1)^{2 r+1}\left(\begin{array}{c}
8 n+1 \\
4 n-k
\end{array}\right)^{a}\left(\begin{array}{c}
4 n+1 \\
2 n-k
\end{array}\right)^{b}\left(\begin{array}{c}
2 n+1 \\
n-k
\end{array}\right)^{c} \equiv 0 \bmod \frac{2(2 n+1)(4 n+1)}{3 n+1}\left(\begin{array}{c}
8 n+1 \\
3 n
\end{array}\right), \\
& \sum_{k=0}^{n} \varepsilon^{k}(2 k+1)^{2 r+1}\left(\begin{array}{c}
8 n+1 \\
4 n-k
\end{array}\right)^{a}\left(\begin{array}{c}
6 n+1 \\
3 n-k
\end{array}\right)^{b}\left(\begin{array}{c}
4 n+1 \\
2 n-k
\end{array}\right)^{c} \equiv 0 \bmod (4 n+1)\left(\begin{array}{c}
8 n+1 \\
3 n
\end{array}\right),
\end{aligned}
$$


Note that

$$
\frac{2}{3 n+1}\left(\begin{array}{c}
8 n+1 \\
3 n
\end{array}\right)=8\left(\begin{array}{c}
8 n+1 \\
3 n
\end{array}\right)-3\left(\begin{array}{c}
8 n+2 \\
3 n+1
\end{array}\right) \in \mathbb{Z}^{+} .
$$

Conjecture 6.17. For all $n, r, s, t \in \mathbb{Z}^{+}$such that $r+s+t \equiv 1(\bmod 2)$, there hold

$$
\begin{array}{rr}
(4 n+1) \sum_{k=0}^{n} \varepsilon^{k} A_{3 n, k}^{r} A_{2 n, k}^{s} A_{n, k}^{t} \equiv 0 & \bmod \frac{1}{6 n+1}\left(\begin{array}{c}
6 n+1 \\
n
\end{array}\right), \\
(4 n+1) \sum_{k=0}^{n} \varepsilon^{k} A_{3 n, k}^{r} A_{2 n, k}^{s} A_{n, k}^{t} \equiv 0 & \bmod \frac{1}{6 n+1}\left(\begin{array}{c}
6 n+1 \\
3 n
\end{array}\right), \\
\sum_{k=0}^{n} \varepsilon^{k} A_{4 n, k}^{r} A_{2 n, k}^{s} A_{n, k}^{t} \equiv 0 & \bmod \frac{1}{8 n+1}\left(\begin{array}{c}
8 n+1 \\
3 n
\end{array}\right), \\
(6 n+1) \sum_{k=0}^{n} \varepsilon^{k} A_{4 n, k}^{r} A_{3 n, k}^{s} A_{2 n, k}^{t} \equiv 0 & \bmod \frac{1}{8 n+1}\left(\begin{array}{c}
8 n+1 \\
3 n
\end{array}\right) .
\end{array}
$$

Finally, for general $m \geq 2$, in (6.6) taking $\left(n_{1}, \ldots, n_{m}\right)$ to be

$$
\begin{cases}(n, n+2, n+4, \ldots, n+m-1, n+m-2, n+m-4, n+m-6, \ldots, n+1), & \text { if } m \text { is odd, } \\ (n+1, n+3, n+5, \ldots, n+m-1, n+m-2, n+m-4, n+m-6, \ldots, n), & \text { if } m \text { is even }\end{cases}
$$

we get the following generalization of congruences (6.1) and (6.7).

Corollary 6.18. Let $m \geq 2$, and let $n, a_{1}, \ldots, a_{m} \in \mathbb{Z}^{+}$and $r \in \mathbb{N}$. Then

$$
\sum_{k=0}^{n} \varepsilon^{k}(2 k+1)^{2 r+1} \prod_{i=1}^{m}\left(\begin{array}{c}
2 n+2 i-1 \\
n+i-k-1
\end{array}\right)^{a_{i}} \equiv 0 \bmod (2 n+2 m-1)\left(\begin{array}{c}
2 n+1 \\
n
\end{array}\right) .
$$

We end this paper with the following challenging conjecture related to Corollary 6.18.

Conjecture 6.19. For all $n, r_{1}, \ldots, r_{m} \in \mathbb{Z}^{+}$such that $r_{1}+\cdots+r_{m} \equiv 1(\bmod 2)$, there holds

$$
\sum_{k=0}^{n} \varepsilon^{k} \prod_{i=1}^{m} A_{n+i-1, k}^{r_{i}} \equiv 0 \quad \bmod \frac{1}{2 n+1}\left(\begin{array}{c}
2 n+1 \\
n
\end{array}\right)
$$

Note that, the $m=1$ case of Conjecture 6.19 is a little weaker than our previous Conjecture 1.3, where the modulus $\frac{1}{2 n+1}\left(\begin{array}{c}2 n+1 \\ n\end{array}\right)$ is replaced by $\left(\begin{array}{c}2 n \\ n\end{array}\right)$. By Corollary 6.18 , it is easy to see that Conjecture 6.19 is true for $n=2$, or $m \leq 6$ and $n=4,9,10,11,3280,7651,7652$ (with the help of MAPLE).

Acknowledgments. This work was partially supported by the Fundamental Research Funds for the Central Universities, Shanghai Rising-Star Program (\#09QA1401700), Shanghai Leading Academic Discipline Project (\#B407), and the National Science Foundation of China (\#10801054).

\section{References}

[1] G. E. Andrews, R. Askey and R. Roy, Special Functions, Encyclopedia of Mathematics and its Applications, Vol. 71, Cambridge University Press, Cambridge, 1999.

[2] N. J. Calkin, Factors of sums of powers of binomial coefficients, Acta Arith. 86 (1998) 17-26.

[3] X. Chen and W. Chu, Moments on Catalan numbers, J. Math. Anal. Appl. 349 (2009) 311-316.

[4] D. Foata and P. Leroux, Polynômes de Jacobi, interprétation combinatoire et fonction génératrice, Proc. Amer. Math. Soc. 87 (1983) 47-53. 
[5] E. Georgiadis, A. Munemasa and H. Tanaka, A note on super Catalan numbers, http://arxiv.org/pdf/1101.1579.

[6] I.M. Gessel, Super ballot numbers, J. Symbolic Comput. 14 (1992) 179-194.

[7] A. Granville, Arithmetic properties of binomial coefficients, I, Binomial coefficients modulo prime powers, in: Organic Mathematics (Burnady, BC, 1995), CMS Conf. Proc., 20, Amer. Math. Soc., Providence, RI, 1997, pp. 253-276.

[8] V. J. W. Guo, F. Jouhet and J. Zeng, Factors of alternating sums of products of binomial and $q$-binomial coefficients, Acta Arith. 127 (2007) 17-31.

[9] V. J. W. Guo and J. Zeng, The number of convex polyominoes and the generating function of Jacobi polynomials, Discrete Appl. Math. 154 (2006) 587-593.

[10] V. J. W. Guo and J. Zeng, Factors of binomial sums from the Catalan triangle, J. Number Theory 130 (2010) $172-186$.

[11] J. M. Gutiérrez, M. A. Hernández, P.J. Miana and N. Romero, New identities in the Catalan triangle, J. Math. Anal. Appl. 341 (2008) 52-61.

[12] P. J. Miana and N. Romero, Moments of combinatorial and Catalan numbers, J. Number Theory 130 (2010) 1876-1887.

[13] E. D. Rainville, Special Functions, Chelsea Publishing Co., Bronx, New York, 1971.

[14] Z.-W. Sun and W. Zhang, Binomial coefficients and the ring of $p$-adic integers, Proc. Amer. Math. Soc. 139 (2011) 1569-1577.

[15] S. O. Warnaar and W. Zudilin, A q-rious positivity, Aequat. Math. 81 (2011) 177-183.

[16] L. L. Zhao, H. Pan and Z.-W. Sun, Some congruences for the second-order Catalan numbers, Proc. Amer. Math. Soc. 138 (2010) 37-46. 